\title{
A Review on Registration of Medical Images Using Graph Theoretic Approaches
}

\author{
Akshaya R, Hema P Menon \\ Department of Computer Science and Engineering, Amrita School of Engineering, Coimbatore Amrita Vishwa \\ Vidyapeetham, India
}

\begin{tabular}{l} 
Article Info \\
\hline Article history: \\
Received Nov 22, 2017 \\
Revised Jan 9, 2018 \\
Accepted Oct 18, 2018 \\
\hline
\end{tabular}

Keywords:

Graph theory

Image registration

Medical images

Non-rigid registration

Rigid registration

\begin{abstract}
Image registration is very essential for image analysis especially in the medical field. The registered image helps us to find a lot of things like the presence of a tumor, aneurysm, and many more. Image registration is a process of aligning two images that were captured during different conditions that makes analyzing of images possible. There are various methods in which images can be registered. This paper mainly discusses the methods that use the graph approach to register the medical images. The main focus is on rigid and non-rigid registration techniques and gives a brief description of the methods present.
\end{abstract}

Copyright $@ 2018$ Institute of Advanced Engineering and Science. All rights reserved.

\section{Corresponding Author:}

Akshaya R,

Department of Computer Science and Engineering,

Amrita School of Engineering,

Coimbatore Amrita Vishwa Vidyapeetham, India.

Email: cb.en.p2cvi16001@cb.students.amrita.edu,p_hema@cb.amrita.edu

\section{INTRODUCTION}

In present day's world, with vast development in the image capturing techniques, images are used in almost all areas from medical industry to space technology. The images that are captured here are not one or two but huge in numbers, if at all we want our computers to detect any changes, identify something from them, then, all must be weighed on the same scale. But the images that are captured are different due to various reasons on the pretext of extracting more information from the images of the object or scene. To analyze these images, first they have to be brought to the same scale, that is, they have to be aligned to each other. This is done by the process of Image registration.

\subsection{Background}

During Image registration, images are mapped to a common co-ordinate system which helps to do analysis on the given set of images thereby becoming an important process in Image processing and analysis. The images captured are different from one another because of the following reasons (i) captured from different Viewpoints and Depth (ii) captured at different timings (iii) using different modes [1], [2]. The images are captured from different Viewpoint in case of a Satellite, the images that are sent are by the satellite are from different Viewpoint as they are moving continuously; If we are monitoring a particular object or a scene then the images that are got are at different timings; In medical images, there are different modes in which the images can be captured, for example the brain images can be got from CT scan or MRI to analyze the condition. The above are the scenarios where we would require Image registration to be analyzed before we make certain conclusions. The major areas where Image registration is used are remote sensing, 
weather monitoring, change detection, Environment monitoring, Medical applications etc., In this paper our major concentration would be on the Medical image registration [3], [4].

Advancements in medical imaging makes it important not only in diagnosis of diseases but also in areas of planning, executing and assessing a surgery. There are wide applications of Image analysis tasks in the field of medicines like Retinal fundus detection, Tumor detection, etc., The various modes that an image can be obtained is Computed Tomography, Magnetic Resonance Imaging, Positron Emission Tomography, Single-Photon Emission Computed Tomography, Functional Magnetic Resonance Imaging, IVUS images or a combination of these sources which is called a multimodal images etc., All these images are used to capture various parts of body like the Brain, Retina, Lungs, Bladder, Breast, Liver, Spine etc., There are a lot of research that is happening in the Image registration [5]-[8].

\section{RESEARCH METHOD}

The process of aligning two or more images is called Image registration. One of the image is considered as the reference image and all the other captured images are aligned with respect to it. We decide on a common co-ordinate system and all the images are arranged in that space. There are numerous ways we can classify the Registration process. They are (i) based on dimensions (ii) based on the nature of transformation (iii) based on the optimization procedures (iv) based on the modes (v) based on the subject (vi) based on the object and many more. Based on dimensions, they can be classified as 2D-2D Registration, 2D-3D Registration or 3D-3D Registration all these are done in the spatial domain whereas images can be registered in time series also. Based on the nature of transformation we can classify them as Rigid, Non-Rigid, Projective, Affine, Curved. Similarly, in case of optimization techniques there are various methods that can be broadly put under categories called the Parameter computed or the Parameter Searched. Mode of the image could be either a single mode or multimode which mostly comes under medical images where we use images from different modes to predict or find something. Objects can be vastly classified, it is actually the object that is being captured, as we are concentrating on medical image registration the objects that come under this are Head (brain, retina, dental, etc.,), Thorax (breast, cardiac, etc.,), Abdomen (liver, kidney, etc.,), Spine and Limbs. The major concentration of the paper is Graph-based methods to solve image registration and the paper mostly deals with Rigid and Non-rigid registration. Registration is an important step not only in medical image analysis but also in fusion, in super resolution, shape analysis, point cloud registration, biometrics and in 3D reconstruction [9]-[14]. Graph method is preferred in case of image registration because of the fact that even if the image is rotated or translated the node in the graph of an image are connected in the same way. Lezoray et al. has explained the concepts of graphs and how graphs used in images [15].

\subsection{Rigid Registration}

Rigid image Registration is very simple as the changes in the images obtained is only translation, rotation, shear, zoom etc. Its only mapping of two or more images. Its assumed that one image remains stationary whereas the other images are spatially transformed. A mapping has to be defined to transform the target image to match the reference image. The parameters of the transform are estimated and the images are transformed using these. In this section, the research work with respect to rigid registration has been discussed.

One of the oldest method used for image registration was proposed by Jasiobedzki Piotr where alignment of images using the adaptive adjacency graphs is done. The technique used in this paper is describing the retinal vascular network that is detected from the reference image to align it with the others. Some of the control points used are the Bifurcations, arteriovenous crossings and vessels. The segmented reference image is the denoted as an Adaptive adjacency graph or the AAG. The AAG contains active contours that are connected in the network. Registration of images is done by placing the adjacency graph of the reference image on the other and allowing the data to adapt to the previous one [16]. Later Sabuncu Mert R., and Peter Ramadge provided a detailed analysis on the use of minimum spanning trees as a tool for alignment of images got from multimodal sources. It uses a graph theoretic method that simultaneously estimates the alignment and the descent direction with the help of transforms. The alignment is measured using the minimum entropy graphs and a descent based optimization. The alignment measure is compared with the plugin entropy estimators which gives us information on the how the data is considered leading to the registration of the image. The algorithm is compared with the other by simulating it into a 3D model. In this method, prior information is combined to avoid problems that occur at the initial stages [17].

Weibel Thomas et al. suggested a method that can reduce the computational time that a graph-cut requires. The nodes from the bladder image, that are in the graph are brought down by computing the spatial properties of an image, which decreases the amount of information that is lost. The reduction of these node 
does not affect the quality of the image registration and the bladder walls are computed using the panoramic maps. The edge value of the graph is found with the help of a watershed transform. The evaluation of the algorithm is done using the Registration error, Euclidean pixel deviation (mean deviation between the source and target image) [18]. Further, Deng Kexin et al. in their paper suggested an algorithm called Graph matching-Iterative closest point to align the images of retina pairwise. The problem of retinal image registration is handled here using Graph Matching. In this method, the features extracted are the retinal vascular features. An undirected graph is constructed and the normalized path is calculated for matching the edges in the graph. It integrates the GM and the ICP algorithm to find out a transformation model. The STRUCTSAC algorithm is applied to it to remove the false matching in the result obtained. The evaluation of the method is based on how salient the vascular graph is and the overall working of the algorithm. The vascular graph is salient based on the following criterions like the average of correctly matched nodes, recall rate and the success of the registration. The parameters used for evaluating the method are the normalized Correlation Coefficient (NCC), normalized Mutual Information (NMI), vessel Centerline Error Measure (CEM) [19]. Lombaert, H., \& Cheriet, F show that the graph-cuts, which mostly has been used to tackle de-noising and finding correspondences separately, can be used simultaneously. This method can be adopted when only one clear image is present and the others are all degraded. The performance of the method is measured using the RMSE value and when it is done together giving a better performance. The method is advantageous when we get a sequence of corrupted images [20].

Lupaşcu Carmen Alina et al. proposes a semi-automatic method for registration of retinal images with extraction of line features connecting the point like bifurcation points, branching's, cross over points, endpoints. A line on the graph indicates that it is a connection in the vascular segment in the retinal image. The landmarks are extracted manually to avoid segmentation errors that can cause loss of data. A straightline model is computed to measure the similarity in the matched lines. The transformation function is calculated from the results that were obtained during matching. Performance of the registration process is computed from the values of cumulative inverse consistency error (CICE) [21]. Parisot et al proposes a sparse method to segment and register the brain tumor by modelling it as a pairwise discrete Markov Random Field model mainly for identification of Gliomas, a type of tumor. A graphical model is used to overlay the sparse grid on to the image domain where its classified and displaced based on the image similarity. Constraints are added to check the smoothness of deformation field. The main drawback of discrete method is the tradeoff between the precision and the complexity which is handled by adding uncertainty in this method. The refinement of the sparse grid reduces the complexity and removal of inactive points helps in the reduction of runtime. The registration of these images was evaluated based on the Dice score, false positive and the true positive rates, MAD was calculated between the source and the floating image [22].

Pinheiro Miguel Et al. presents a technique where the calculation of path descriptors in geometrical graphs are done to find the similarity between them. It mainly aims in creating a method that can be applied where standard methods cannot be applied that is where the image appearance is different, unique key points cannot be identified or there is not much of texture. The main aim is to calculate the path descriptors to match two graphs. The parameters used for evaluating the method with the state of art methods, are Euclidian distance between the registered graphs and their processing time [23]. Menon Hema P., Et al. illustrates a method for selecting the points for finding correspondences based on the structural information of the image as it remains the same even when the illumination in the image changes. The method proposed is called Vertex Degree Neighborhood (VDN). Here the initial match is found using the degree and the neighbors of the node. Then its improved by feature matching using features like the entropy, mean and standard deviation. The initial points were got using the Harris corner detector and graph is built using Delaunay triangulation. The parameter of comparison is Target Registration Error [24]. Zhong Yang-jun and Lan-Zhen Chen, Et al. presents the registration of mammogram images using SIFT and graph transformation. Feature points are derived from the image and GTM is mainly done to obtain accurate results as it minimizes the incorrect matches. The registration is done using the Thin-plate spline (TPS) interpolation. The evaluation is done based on the correlation coefficient (CC), the sum of squared differences (SSD) and the visual effect [25].

Czajkowska Joanna Et al. proposed a method for registration that uses path similarity skeleton graph matching along with weight cliques to achieve matching. This method allows us to match the voxels from different CT series. The main application of this method can be found in detecting abdominal aortic aneurysm by visualizing the pre- and post-operative CT data. The method compares two algorithms applied for 3-D registration that does a segmentation of the aorta and internal organ using graph approaches. The analysis was carried out on real CTA data of the patients having abdominal aortic aneurysm [26]. C. Leng et al. proposes a sparse based matrix factorization method called the graph regularized non-negative matrix factorization. Using graph regularization and total variation will help us preserve the intrinsic details of the image and the small details respectively. The features are extracted using the extraction algorithms like 
the Harris corner detector. The performance of the algorithm is compared with other methods like the Zass's and the Caeill's method where the computation time and accuracy of the algorithm is better the other two. The discriminating ability of the algorithm is calculated using the root mean square error value [27]. Chen $\mathrm{Li}$ Et al. proposed the method where the use of network structure and circuit simulation is done to register the given images. Vessel images were transformed into graphs and their segmentation is done to reduce the complexity of the calculation. Weighted graphs were converted into circuits and registration was done based on the node voltages. It efficiently handles the problem of matching large number of vascular images. First the salient points are identified and a circuit conversion model is proposed where the spatial difference is reflected as the node voltage. Network decomposition is done using the NSI method and the branching criteria is used to deal with large scale networks [28]. Parekar et al. proposed a method for retinal image registration by matching the bifurcations in the image. A fully connected vascular network is got with the help of Dijkstra's algorithm. The images are subjected to preprocessing steps as false matches are to be removed. The proposed method is invariant to translation, rotation, scaling as only normalized features are used. Order statistic filters are used for processing initially, followed by multi-scale ridge detection and finally Dijkstra's for joining the broken edges. Pre-processing steps consist of noise removal, non-maximal suppression etc. The parameters for evaluation are mean and standard deviation of the registration error [29]. Zhang et al. proposes a method that makes use of Delaunay Triangulation. The images used for experimentation are multispectral and panoramic images the feature points are extracted using SIFT algorithm and the Delaunay Triangulation to remove the false matches. Correspondences are established using the Delaunay triangulations. The Algorithm removes the false matches and has good speed and accuracy [30].

\subsection{Non-Rigid Image Registration}

Our body is not always rigid, there are parts in our body that do not remain stationary say our heart, deformations in our face etc., Registration of such images are done using non-rigid image registration. The transformation functions used here are non-linear like the spline, elastic model etc. [31]. The algorithms that help us in registering such images are discussed. One of the oldest algorithm found in non-rigid image registration was by Fischler and Elschlager to register the deformation in face [32]. Further, Tang Tommy W $\mathrm{H}$ et al. proposes a method where the non-rigid image registration is considered as a discrete labelling problem. Pixels in the source image is assigned with a displacement vector that denotes its spatial position in the floating image. The optimization of this method uses the graph-cuts method via alpha expansions. The sharp changes in the displacement labels is penalized using a first derivative smoothness constraint. Using of graph-cuts ensure the finding of the minima. The method is accurate and robust when compared with the rest of the state of art methods. The parameter that is used to assess the performance is the overlap measure and was found to better than the FFD and demons algorithms [33]. Further, Ronald WK, and Albert CS Chung proposed a method to solve the problem of non-rigid registration using graph-cuts. This paper handles a multi-level non-rigid registration, where the deformation field of one level is passed on to the next. Using this approach, the number of levels in the problem is greatly reduced thereby the speed of registration is increased. The performance is evaluated based on the computational time and is compared with DEMONS and the FFD algorithms and the single level registration [34]. R. W. K. So and A. C. S. Chung proposed an improvement to the learning-based similarity measure for non-rigid image registration using the KLD which uses the prior knowledge of the joint distribution of the intensities of the pre-aligned images. The new formulation is the use of Markov random field (MRF) with KLD and graph-cuts. The valuation is based on sum of absolute difference and mutual information. It is compared with Free form deformation (FFD) and DEMONS algorithm [35].

Ronald WK et al. proposes a graph-cuts based method for registration of non-rigid images where the registration is regarded as a discrete labelling problem. The deformation model used in this case is flexible and non-parametric. In the registration process, each pixel is allocated three labels and this the displacement vector that specifies the position in the floating image. A first order based smoothness based constraint is used to remove the sharp changes in the adjacent displacement labels. The optimization of the registration process is done using graph-cuts method which uses (alpha)-expansion to reach a local or a global minimum. The algorithm is compared with the existing methods like the DEMONS based method, linear programming method, free-form deformation based method. The method is evaluated using parameters like the overlap measure, distribution of absolute intensity errors. The algorithm is robust against all challenges like huge deformation, ripple distortion etc., [36]. Chowdhury, Ananda S Et al. have improved the performance of a non-rigid image registration with usage of data term. This can be helpful in computational neuroanatomy where we can detect the conditions like schizophrenia, registration of retinal images etc. There are several advantages in this method like the displacement terms can be assigned from the data term. It acts as a strict penalty on mismatches and the data term can handle the dissimilarity in the intensity patterns due to 
illumination changes in the images. The work is done on the MRI images of the brain and the retinal images. The results are compared to that of the demons algorithm using the absolute intensity differences for registration accuracy [37]. A. Szmul, B Et al. proposes a method that uses graph-cuts as an efficient optimization tool. Here the image is represented as a graph constructed from the sparse super-voxels combined with graph-cuts. Using the sliding motion modelling, a relaxed graph is constructed which helps in estimation of deformation. Registration is achieved using image-guided filter in the sparse deformation field. The experiments are done on CT lung dataset that is received from a single mode and its intra-patient. It is mainly applied in radio therapy planning, monitoring of treatment, and ventilation quantification. The method was evaluated based on the target registration error which is found to be better than the demons algorithm [38]. Serradell et al. proposes a method that does not rely only on local feature similarity. This method does not require initial matches and can do some partial matching. Non-linear deformations are handled with the help of Gaussian Process. Even in case the information is missing the correspondences are matched using an iterative process. The experiment is conducted on both synthetic images and the angiography images. The number of correspondences is reduced in this method without the use of any deformation model. First a coarse alignment is done following which a finer method is used [39]. Chen et al proposes a method that is a combination of non-rigid image registration and semi-automatic segmentation of liver that is used for a guided surgery. This method puts k-means clustering and graph cuts together to develop a robust algorithm. A free form deformation is applied to register these images. Gaussian mixture modelling is used for intensity distribution of the liver images. The statistical parameters from the image is calculated using the prior knowledge available. Thresholding is applied to find out the candidate points from the liver and then k-means algorithm is used to find out the non-liver pixels. The seeds are obtained from the previous step and the segmentation is based on graph-cuts method. The segmentation and the registration are combined together which is used during invasive surgeries. They use both CT and MR images for the process. The parameters that are used for validation of the algorithm is average registration error and the processing speed [40]. El-Baz et al proposes a method for registration of Lung nodules. This method helps us identify lung nodules that may later lead to cancer, this helps us in early detection. We monitor the lung nodules in a successive manner from the CT scan of the patient. The process starts with extraction of the lung regions by modelling its gray level intensities, followed by classification of parts of the lung, applying rigid registration and the compensating for the heart beats and breathing. The process uses the normalized mutual information in the final steps. To check whether the nodes have grown or not re-slicing the image [41].

\subsection{Group-Wise Image Registration}

Group-wise image Registration mostly aims in registering a set of images into a common space instead of registering it to a particular image separately. Research has been done in this area and the outcome are the following. S. Ying, G. Et al. proposes a method for group registration of the different groups of images. The method uses a two-level hierarchical graph to model the problem by using intra graph for the images in a group and inter graph for images belonging to different groups. The evaluation of the algorithm is done by calculating the dice ratio. The ratio achieved by performing this on the images is better than the existing methods. The algorithm preserves the topology of the images during the process of registration [42]. S. Ying Et al. investigates a method where the group images are registered without the use of the reference image avoiding the bias in the registration process. The images are modelled using graphs where the nodes are the images and the edges are the geodesic path between them. Registration is achieved using the dynamic shrinking problem until the nodes are close to each other. The topology of the image is preserved in the process. All the images are warped simultaneously towards the center eliminating the possibility of bias. The parameter for assessing it is Dice ratio [43]. Tang Zhenyu Et al. presents a technique that accurately registers the similar images to the group center using the shortest path. The similarity measure is built on sparse directed graphs (digraph), where the nodes are connected by shortest distances. The registration is attained by iterative method where the large deformation field is split into series of smaller ones. Results suggest that the method is robust and achieves better accuracy when compared with existing methods. The performance is evaluated by the overlap region measured using Jaccard index [44].

Graph-based methods are not only used for medical images, they are also used in various other areas and a few of them are discussed below. M. Izadi and P. Saeedi, present an algorithm where the correspondences are matched accurately. The feature points extracted are used to find out the geometrical relationship in the graph. It starts matching the points in the image and graph is created for the point and knearest neighbors. The angular distances are used to find the matching points in the second image. The advantage of this method is its capability to handle outliers. The evaluation parameters are Accuracy, Precision, Recall, and Specificity [45]. Leng Et al. proposes a computation technique that is based on the projection theorem of energy conservation to improve the image registration performance. First the intergraph is constructed based on the proximity and from that and intra-graph using the node sets. Then the 
projection theorem is applied to project that data into a lower dimension space. Analytical methods are used to find the correspondences in the image which is very accurate even when there are lot of nodes in it. The accuracy of this method is compared with the state of art methods [46]. Zhu Et al. presents a method to register the color image and depth information. The correspondences between the color image and depth information is found using a contour based approach. The correspondences on the contours will have similar shape descriptors and this helps us use Munkres algorithm to solve the bipartite graph. The similarity is computed based on the chi-square method and the transform model is estimated using the RANSAC method. The method is compared against existing methods like COPAP, ACO and Hungarian with parameters like the run time and the RMSE values [47].

Ngo Et al. proposes an algorithm where the parameter space of the rigid transform is locally computed using the gradient decent paradigms. The parameter space has three dimensions and the overall space is subdivided as a subset of R3 having a polynomial complexity. Here the graph is integrated with the descent scheme that will lead to the minimum which solves the problem of image registration. The optimization process uses a multi-scale approach to speed it up. The parameters used for validation are the run time complexity, distance based on signed function and the average degree of DRT graphs [48]. Zakharov Et al. considers the problem of point matching and main objective is to eliminate the false correspondences. Firstly, Scott and Longuet-Higgins algorithm is used and a graph is constructed based the features extracted from these images. Singular value decomposition is done and the correspondences are found using the descriptor SURF. This algorithm helps us to find the significant false correspondences. The speed of the algorithm is also high. It's mainly used in three-dimensional reconstruction tasks. The algorithm is compared with RANSAC [49]. Sanromà Et al. proposes a method for finding the point-set correspondences using the graph matching method which is modeled as a mixture modelling problem which accounts for both structural and the geometrical parts. Graph matching is approximated using the Expectation-maximization algorithm. The corresponding continuous variables are solved using Soft-assign, imposing a constraint and also controls the level of discretization. Effective mechanisms are used detect and remove outliers. The evaluation of the algorithm is done using registration accuracy and recognition ability [50]. Zhang Et al. proposes a graph spectral method that uses isomeric projections. This is mainly done to solve the problem of matching the feature points using the structural information that is got from the graph. Using this projection, the graphs are projected to a matrix that has the normalized values. The projection of data is unique and thus can be used for computing the correspondences. A Laplacian matrix is used to preserve the information that is related to the position of a point. The graph matching problem is handled using the structural and positional information. The results got show that the algorithm is accurate and its robust [51]. Gul et al. proposes a method for the registration of brain images where features are extracted using SURF and the matching is done using Euclidean measure. The false matches that are present in the image is removed using the R-RANSAC algorithm along with the SPRT to lessen the runtime. Final registration of the image is done using the least squares method. The algorithm is robust and efficient due to the combination of SURF and R-RANSAC. The algorithm is fast as well as accurate when compared with the other methods [52]. Zhang et al. proposes a method of registration that uses wavelet transform. The registration takes place as two steps one the coarse registration and the other is the fine registration. The parameters of registration are got through the ant colony search method. The algorithm is robust to noise, has high accuracy and fast in computation [53].

\section{RESULTS AND ANALYSIS}

This section gives a summary of the results obtained by various researchers for registration off medical images using graph based approaches. Based on the various parameters that have been used for assessing the quality of the output, the methods and sample outputs are shown in Table 1. 
Table 1. Summary of Registration on medical images

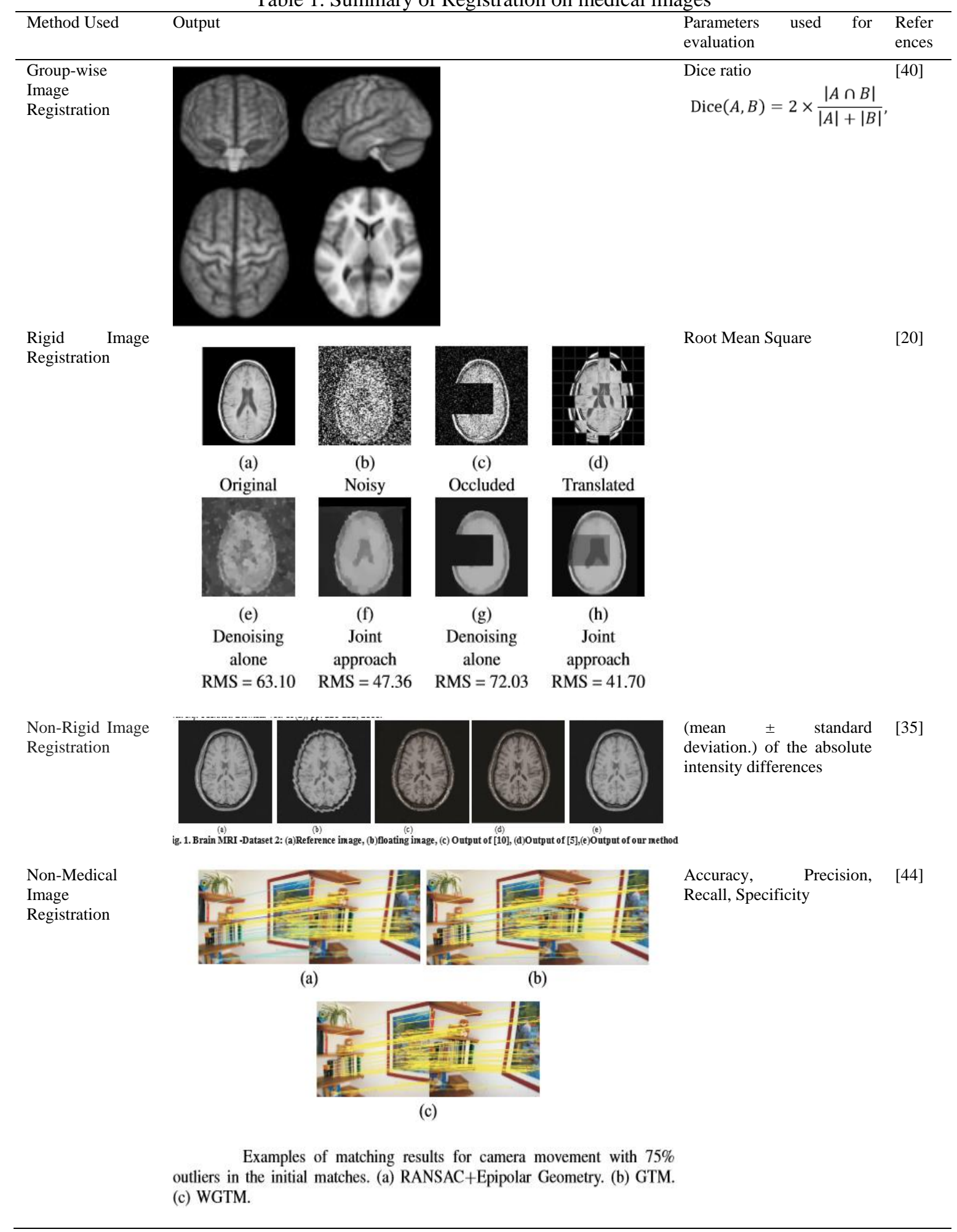

\subsection{Observation and Inferences}

a) Graph based methods are mainly based on pixels.

b) Time taken for the process is directly proportional to the number of nodes or edges present in the graph i.e., as the nodes increase the time taken also increases. 
c) The values of the edges are based on the intensity or the distance between the pixels.

d) Selection of points as nodes is a very crucial step in graph based methods.

e) The number of points in both of the images should be the same and it should be homologous.

f) Hence, correspondence matching between these points has also gained importance.

\section{CONCLUSION}

A lot of Research has been done in the area of image registration and the it is still going on. It becomes very important to have a good registration algorithm as it is the first step to analyzing something. Rigid Registration has quite good amount of research as it is easy to formulate when compared to the nonrigid registration. And most work take place in areas like brain and retina as the data is available, but the problem with this is that real tumor-patients data is really difficult to get. Hence most of the works take place using templates which is more or less perfect and does not have any error like error that occur during capturing of patient's data. Non-rigid image registration on the other hand is still challenging due to the smoothness requirement and the huge degree of freedom. This paper mainly aims at introducing methods that are developed to solve image registration problem using graph approaches.

\section{REFERENCES}

[1] Zitova B, Flusser J. Image registration methods: a survey. Image and vision computing. 2003 Oct 31; 21(11): 977-1000.

[2] Brown LG. A survey of image registration techniques. ACM computing surveys (CSUR). 1992 Dec 1; 24(4): 32576.

[3] Granero MA, Baroni MP, Costa ET, Radeva PI. An alternative technique for Imaging Registration in IVUS images. In Computational Science and Computational Intelligence (CSCI), 2015 International Conference on 2015 Dec 7 (pp. 464-469) IEEE.

[4] Wyawahare MV, Patil PM, Abhyankar HK. Image registration techniques: an overview. International Journal of Signal Processing, Image Processing and Pattern Recognition. 2009 Sep;2(3):11-28.

[5] Hill DL, Batchelor PG, Holden M, Hawkes DJ. Medical image registration. Physics in medicine and biology. 2001 Mar;46(3): R1.

[6] Rueckert, Daniel, and Julia A. Schnabel. Medical image registration. In Biomedical Image Processing, pp. 131-154. Springer Berlin Heidelberg, 2010.

[7] Zhang S, Zhi L. Multi-modality image registration with gradient orientation information based on entropic spanning graph. In Information Science and Technology (ICIST), 2017 Seventh International Conference on 2017 Apr 16 (pp. 470-473). IEEE.

[8] Mani VR. Survey of medical image registration. Journal of Biomedical Engineering and Technology. 2013 Jan 23;1(2):8-25.

[9] Menon HP, Narayanankutty KA. Comparative performance of different perceptual contrast fusion techniques using MLS. International Journal of Biomedical Engineering and Technology. 2015;18(1):52-71.

[10] R. Shwetha and Rajathilagam B. Super resolution of mammograms for breast cancer detection. International Journal of Applied Engineering Research. 2015; 10(1):21453-21465.

[11] Zeng W, Yang YJ, Razib M. Graph-Constrained Surface Registration Based on Tutte Embedding. In Proceedings of the IEEE Conference on Computer Vision and Pattern Recognition Workshops 2016 (pp. 76-83).

[12] Huang X, Zhang J, Fan L, Wu Q, Yuan C. A Systematic Approach for Cross-source Point Cloud Registration by Preserving Macro and Micro Structures. IEEE Transactions on Image Processing. 2017 Apr 19.

[13] Ong EP, Xu Y, Wong DW, Liu J. Retina verification using a combined points and edges approach. In Image Processing (ICIP), 2015 IEEE International Conference on 2015 Sep 27 (pp. 2720-2724). IEEE.

[14] Arathi. T. and Latha Parameswaran. Image Reconstruction from 2D stack of MRI/CT to 3D using Shapelets. International Journal of Engineering and Technology (IJET). 2014;6(1):2595 - 2603.

[15] Lézoray O, Grady L. Graph theory concepts and definitions used in image processing and analysis. Image processing and analysis with graphs: theory and practice. CRC Press, Boca Raton, FL. 2012:1-24.

[16] Jasiobedzki P. Registration of retinal images using adaptive adjacency graphs. In Computer-Based Medical Systems, 1993. Proceedings of Sixth Annual IEEE Symposium on 1993 Jun 13 (pp. 40-45). IEEE.

[17] Sabuncu MR, Ramadge P. Using spanning graphs for efficient image registration. IEEE Transactions on Image Processing. 2008 May;17(5):788-97.

[18] Weibel T, Daul C, Wolf D, Rösch R, Ben-Hamadou A. Endoscopic bladder image registration using sparse graph cuts. In Image Processing (ICIP), 2010 17th IEEE International Conference on 2010 Sep 26 (pp. 157-160). IEEE.

[19] Deng K, Tian J, Zheng J, Zhang X, Dai X, Xu M. Retinal fundus image registration via vascular structure graph matching. Journal of Biomedical Imaging. 2010 Jan 1;2010:14.

[20] Lombaert H, Cheriet F. Simultaneous image de-noising and registration using graph cuts: Application to corrupted medical images. In Information Science, Signal Processing and their Applications (ISSPA), 2012 11th International Conference on 2012 Jul 2 (pp. 264-268). IEEE. 
[21] Lupaşcu CA, Tegolo D, Bellavia F, Valenti C. Semi-automatic registration of retinal images based on line matching approach. In Computer-Based Medical Systems (CBMS), 2013 IEEE 26th International Symposium on 2013 Jun 20 (pp. 453-456). IEEE.

[22] Parisot S, Wells W, Chemouny S, Duffau H, Paragios N. Concurrent tumor segmentation and registration with uncertainty-based sparse non-uniform graphs. Medical image analysis. 2014 May 31;18(4):647-59.

[23] Pinheiro MA, Kybic J. Path descriptors for geometric graph matching and registration. In International Conference Image Analysis and Recognition 2014 Oct 22 (pp. 3-11). Springer, Cham.

[24] Menon HP, Narayanankutty KA, Indulekha TS. Feature Point Selection using Structural Graph Matching for MLS based Image Registration. International Journal of Computer Applications. 2014 Jan 1;100(4).

[25] Zhong YJ, Chen LZ. A novel algorithm based on SIFT and Graph transformation for mammogram registration. In Proceedings of the 2012 International Conference on Cybernetics and Informatics 2014 (pp. 1897-1903). Springer, New York, NY.

[26] Czajkowska J, Feinen C, Grzegorzek M, Raspe M, Wickenhöfer R. Skeleton Graph Matching vs. Maximum Weight Cliques aorta registration techniques. Computerized Medical Imaging and Graphics. 2015 Dec 31;46:142-52.

[27] Leng C, Chen Z, Cai G, Cheng I, Xiong Z, Tian J, Basu A. Total variation constrained graph regularized NMF for medical image registration. In Image, Video, and Multidimensional Signal Processing Workshop (IVMSP), 2016 IEEE 12th 2016 Jul 11 (pp. 1-5). IEEE.

[28] Chen L, Lian Y, Guo Y, Wang Y, Hatsukami TS, Pimentel K, Balu N, Yuan C. A vascular image registration method based on network structure and circuit simulation. BMC bioinformatics. 2017 May 2;18(1):229.

[29] Parekar J, Porwal P, Kokare M. Automatic retinal image registration using fully connected vascular tree. In Signal and Information Processing (IConSIP), International Conference on 2016 Oct 6 (pp. 1-5). IEEE.

[30] Yongmei Zhang, Li Ma1,2, Rui Zhang. A Quick Image Registration Algorithm Based on Delaunay Triangulation. TELKOMNIKA, 2013 February, 11(2):761 773.

[31] Crum WR, Hartkens T, Hill DL. Non-rigid image registration: theory and practice. The British journal of radiology. 2004 Dec;77(suppl_2):S140-53.

[32] Fischler MA, Elschlager RA. The representation and matching of pictorial structures. IEEE Transactions on computers. 1973 Jan;100(1):67-92.

[33] Tang TW, Chung AC. Non-rigid image registration using graph-cuts. In International Conference on Medical Image Computing and Computer-Assisted Intervention 2007 Oct 29 (pp. 916-924). Springer, Berlin, Heidelberg.

[34] So RW, Chung AC. Multi-level non-rigid image registration using graph-cuts. In Acoustics, Speech and Signal Processing, 2009. ICASSP 2009. IEEE International Conference on 2009 Apr 19 (pp. 397-400). IEEE.

[35] So RW, Chung AC. Non-rigid image registration by using graph-cuts with mutual information. In Image Processing (ICIP), 2010 17th IEEE International Conference on 2010 Sep 26 (pp. 4429-4432). IEEE.

[36] So RW, Tang TW, Chung AC. Non-rigid image registration of brain magnetic resonance images using graph-cuts. Pattern Recognition. 2011 Nov 30;44(10):2450-67.

[37] Chowdhury AS, Roy R, Bose SK, Khalifa F, Elnakib A, El-Baz A. Non-rigid biomedical image registration using graph cuts with a novel data term. In Biomedical Imaging (ISBI), 2012 9th IEEE International Symposium on 2012 May 2 (pp. 446-449). IEEE.

[38] Szmul A, Papiez BW, Bates R, Hallack A, Schnabel JA, Grau V. Graph Cuts-Based Registration Revisited: A Novel Approach for Lung Image Registration Using Supervoxels and Image-Guided Filtering. In Proceedings of the IEEE Conference on Computer Vision and Pattern Recognition Workshops 2016 (pp. 152-159).

[39] Serradell E, Glowacki P, Kybic J, Moreno-Noguer F, Fua P. Robust non-rigid registration of $2 D$ and $3 D$ graphs. In Computer Vision and Pattern Recognition (CVPR), 2012 IEEE Conference on 2012 Jun 16 (pp. 996-1003). IEEE.

[40] Chen YW, Tsubokawa K, Foruzan AH, Morikaw S, Kurumi Y. Image segmentation and registration techniques for MR-Guided Liver Cancer Surgery. In Mechatronics and Embedded Systems and Applications (MESA), 2012 IEEE/ASME International Conference on 2012 Jul 8 (pp. 105-108). IEEE.

[41] El-Baz A, Yuksel SE, Elshazly S, Farag AA. Non-rigid registration techniques for automatic follow-up of lung nodules. In International Congress Series 2005 May 31 (Vol. 1281, pp. 1115-1120). Elsevier.

[42] Ying S, Wu G, Liao S, Shen D. Inter-group image registration by hierarchical graph shrinkage. In Biomedical Imaging (ISBI), 2013 IEEE 10th International Symposium on 2013 Apr 7 (pp. 1030-1033). IEEE.

[43] Ying S, Wu G, Wang Q, Shen D. Groupwise registration via graph shrinkage on the image manifold. In Proceedings of the IEEE Conference on Computer Vision and Pattern Recognition 2013 (pp. 2323-2330).

[44] Tang Z, Jiang D, Fan Y. Image registration based on dynamic directed graphs with groupwise image similarity. In Biomedical Imaging (ISBI), 2013 IEEE 10th International Symposium on 2013 Apr 7 (pp. 492-495). IEEE.

[45] Izadi M, Saeedi P. Robust weighted graph transformation matching for rigid and nonrigid image registration. IEEE Transactions on image processing. 2012 Oct;21(10):4369-82.

[46] Leng C, Xu W, Li M, Rossol N, He L, Liu D. Image registration based on the projection theorem of energy conservation in graphs. In Fuzzy Systems and Knowledge Discovery (FSKD), 2011 Eighth International Conference on 2011 Jul 26 (Vol. 3, pp. 1976-1980). IEEE.

[47] Zhu Q, Wen T, Xie Y, Gu J, Wang L. Contour-Based Image Registration using Bipartite Graph Matching with Munkres Algorithm. Applied Mathematics \& Information Sciences. 2014 Jan 1;8(1):263.

[48] Ngo P, Kenmochi Y, Sugimoto A, Talbot H, Passat N. Discrete rigid registration: A local graph-search approach. Discrete Applied Mathematics. 2017 Jan 10;216:461-81. 
[49] Zakharov AA, Tuzhilkin AY, Zhiznyakov AL. Finding Correspondences Between Images using Descriptors and Graphs. Procedia Engineering. 2015 Jan 1;129:391-6.

[50] Sanromà G, Alquézar R, Serratosa F. A new graph matching method for point-set correspondence using the EM algorithm and Softassign. Computer vision and image understanding. 2012 Feb 29;116(2):292-304.

[51] Zhang Z, Tian Z. Image registration using isometric projection of graph. In Information Engineering and Computer Science, 2009. ICIECS 2009. International Conference on 2009 Dec 19 (pp. 1-4). IEEE.

[52] Zongyun Gu1, Li Cai, Yunxia Yin, Yatao Ding, Hongxing Kan. Registration of Brain Medical Images Based on SURF Algorithm and R-RANSAC Algorithm. TELKOMNIKA Indonesian Journal of Electrical Engineering, 2014 March, 12(3): $2290 \sim 2297$.

[53] Dapeng Zhang, Jiayan Li. Image Registration Method Based on Wavelet Transform and Ant Colony Optimization. TELKOMNIKA, 2015 June, 13(2):604 613. 